CYBERNETICS AND INFORMATION TECHNOLOGIES • Volume 16, No 5

Special Issue on Application of Advanced Computing and Simulation in Information Systems

Sofia $\bullet 2016$

Print ISSN: 1311-9702; Online ISSN: 1314-4081

DOI: 10.1515/cait-2016-0059

\title{
A New Localization Algorithm Based on Taylor Series Expansion for NLOS Environment
}

\author{
Jin Ren, Jingxing Chen, Wenle Bai
}

School of Electronic and Information Engineering, North China University of Technology, No 5 Jinyuanzhuang Road, Shijingshan District, Beijing, China

Email:rj@ncut.edu.cn

\begin{abstract}
In Non-Line-Of-Sight (NLOS) environment, location accuracy of Taylorseries expansion location algorithm degrades greatly. A new Taylor-series expansion location algorithm based on self-adaptive Radial-Basis-Function (RBF) neural network is proposed in this paper, which can reduce the impact on the positioning accuracy of NLOS effectively on the basis of the measurement error correction. RBF neural network has a faster learning characteristic and the ability of approximate arbitrary nonlinear mapping. In the process of studying, RBF neural network adjusts to the quantity of the nodes according to corresponding additive strategy and removing strategy. The newly-formed network has a simple structure with high accuracy and better adaptive ability. After correcting the error, reuse Taylor series expansion location algorithm for positioning. The simulation results indicate that the proposed algorithm has high location accuracy, the performance is better than RBF-Taylor algorithm, LS-Taylor algorithm, Chan algorithm and LS algorithm in NLOS environment.
\end{abstract}

Keywords: Position location, NLOS propagation, Adaptive processing, RBF neural network.

\section{Introduction}

In recent years, with the rapid development of cellular mobile communication technology, mobile data has been heavily promoted for business development, where various application of wireless location technology, based on user's location also showed its potential, and mobile positioning technology has become a hot field of research. Wireless communication basic positioning techniques including field intensity method, based on Time Difference Of Arrival (TDOA), Time Of Arrivals from (TOA) and Angle Of Arrival (AOA) and various hybrid positioning method [1]. TDOA techniques are open to less equipment modification and don't need 
Mobile Station (MS) with a strict precise time synchronization between base Station and more extensive applications. Taylor series expansion method [2, 3], Chan and Least Squares (LS) algorithms are all based on TDOA location algorithm. The influence of Non-Line-Of-Sight (NLOS) largely affects the positioning accuracy, the additional delay is caused by the multipath error, so performances of algorithms are very big effect. LS algorithm does not need to consider the statistical properties of the error, location accuracy of which in NLOS environment is slightly better than Chan algorithm. Taylor series expansion method need a location near to the actual initial estimate of the position to ensure the convergence of algorithm, and positioning accuracy is higher than that Chan algorithm and LS algorithm in NLOS environment. But Taylor algorithm positioning accuracy in NLOS environment is far below location accuracy under the environment of LOS. So how to reduce the TDOA measurements of NLOS error becomes the key to Taylor algorithm applied in the NLOS environment.

In recent years, the research found RBF (Radial Basis Function, RBF) neural network [5] is a typical forward neural network, which has a simple structure, the advantages of fast learning speed, which has been widely applied on the Function approximation, system identification, pattern recognition and other fields. The RBF network is joined the adaptive adjustment strategy [6,7], in the process of adjustment, according to the distribution of the errors in the input space and the contributions of each RBF to the network, with adaptive adding and removing the number of RBF, and appropriately adjust the center vector. Thus, the network structure always keeps it simple.

\section{Algorithm description}

In NLOS environment, the TDOA measurement error is bigger, having larger influence on Taylor positioning performance of the algorithm, using the characteristics of adaptive RBF neural network to modify TDOA measurement data, which can reduce the NLOS error of TDOA measurement data. Then on the basis of the revised data by Taylor series algorithm to locate, can achieve better location performance. The proposed algorithm is based on this method.

\subsection{Taylor series expansion localization algorithm}

Set $\left(x_{i}, y_{i}\right)$ is for the base station coordinates, $(x, y)$ is the mobile station coordinates under test, $R_{i 1}$ is the distance between the mobile station and the $i$ th base station, $N$ is the number of base station location to participate, $R_{i}$ is the distance between the initial position of the mobile station and the $i$ th base station, $c$ is the speed of light, $\Delta \tau_{i 1}$ is the time lag of signal arriving between the measured service base station $\mathrm{BS}_{1}$ and the $i$-th base station.

Cellular networks based on TDOA location technology in multiple TDOA measurements, can build positioning system of equations

$$
\begin{gathered}
R_{i, 1}=c \Delta \tau_{i, 1}=R_{i}-R_{1}=\sqrt{\left(X_{i}-x\right)^{2}+\left(Y_{i}-y\right)^{2}}-\sqrt{\left(X_{1}-x\right)^{2}+\left(Y_{1}-y\right)^{2}}, \\
i=2, \ldots, N .
\end{gathered}
$$


To solve the nonlinear equations is equivalent to unconstrained nonlinear optimization problems, so must be linearized processing on it firstly.

For a set of TDOA measurements, according to the selected initial coordinates $\left(x_{0}, y_{0}\right)$, Taylor series expansion of Equation (1) and ignore the second order or more weight, is ignored, we can get

$$
\boldsymbol{h}=\boldsymbol{G} \beta+\varepsilon
$$

where

$$
\boldsymbol{\beta}=\left[\begin{array}{c}
\Delta x \\
\Delta y
\end{array}\right], \boldsymbol{h}=\left[\begin{array}{c}
R_{2,1}-\left(R_{2}-R_{1}\right) \\
R_{3,1}-\left(R_{3}-R_{1}\right) \\
\vdots \\
R_{N, 1}-\left(R_{N}-R_{1}\right)
\end{array}\right], \boldsymbol{G}=\left[\begin{array}{cc}
\frac{X_{1}-x_{0}}{R_{1}}-\frac{X_{2}-x_{0}}{R_{2}} & \frac{Y_{1}-y_{0}}{R_{1}}-\frac{Y_{2}-y_{0}}{R_{2}} \\
\frac{X_{1}-x_{0}}{R_{1}}-\frac{X_{3}-x_{0}}{R_{3}} & \frac{Y_{1}-y_{0}}{R_{1}}-\frac{X_{3}-y_{0}}{R_{3}} \\
\frac{X_{1}-x_{0}}{R_{1}}-\frac{X_{N}-x_{0}}{R_{N}} & \frac{Y_{1}-y_{0}}{R_{1}}-\frac{Y_{N}-y_{0}}{R_{N}}
\end{array}\right] .
$$

The Equation (2) by using Weighted Least Square algorithm (WLS), can get least squares estimate solution of $\beta$ is:

$$
\boldsymbol{\beta}=\left(\boldsymbol{G}^{\mathrm{T}} \boldsymbol{Q}^{-1} \boldsymbol{G}\right)^{-1} \boldsymbol{G}^{\mathrm{T}} \boldsymbol{Q}^{-1} \boldsymbol{h} .
$$

In the initial iterations, make $x=x_{0}, y=y_{0}$ can be obtained $R_{i}$ and $G$. In each of the next process will be the last $\beta$ to the current mobile station coordinates $\left(x_{k}, y_{k}\right)$ and repeat the process until $\beta$ small enough. The threshold is $\delta$ namely. When $|\Delta x|+|\Delta y|<\delta$, the iteration process is end. At this time to get the coordinates of the estimates for the MS coordinates $(x, y)$.

\subsection{Taylor series expansion location algorithm based on adaptive RBF}

Measure the TDOA value first, and then through adaptive RBF network modification, finally use Taylor series expansion for positioning.

Specific steps are as follows:

(1) Measured a group of TDOA measurements in NLOS environment, and established adaptive RBF network for error correction training, with the mobile station no containing NLOS error of TDOA measurements for target vectors of neural network training samples.

(2) Used trained adaptive RBF network to simulate TDOA measurements.

(3) The revised TDOA value of Taylor algorithm is used to estimate position.

\section{Adaptive RBF neural network for TDOA measurements under NLOS correction}

Actual channel, as a result of the existence of various obstacles makes waves, which cannot transmitted along a straight line, after reflection and diffraction reach the receiver, so that the influence of system measurement error is bigger, which can be achieved by adaptive RBF neural network for error correction being close to LOS error of the environment. Relative to the RBF neural network, adaptive network has more advantages. 


\subsection{RBF neural network}

RBF network [8] can approximate any nonlinear function, and can handle the difficult parsing the regularity in the system, which has good generalization ability. In nonlinear function approximation, time series analysis, information processing, pattern recognition and other fields, RBF network has been widely used.

Seven stations established NLOS environment provided by TDOA measurements revised adaptive RBF neural network model, RBF network consists of input layer, hidden layer and output layer.

The input layer by seven related base station provided by the six TDOA measurements. Input vector is:

$\boldsymbol{x}=\left[x_{1}, x_{2}, x_{3}, x_{4}, x_{5}, x_{6}\right]=[\mathrm{TDOA} 21$, TDOA31, TDOA41, TDOA51, TDOA61, TDOA71], the role of hidden layer nodes function is to the input signal in the partial response, when the basis function of the input signal near the central range, and hidden layer nodes will produce the larger output, so the network has good local approximation ability. The choice of basis function is gaussian function:

$$
\varphi_{j}=\exp \left(-\frac{\left\|\boldsymbol{x}-\boldsymbol{c}_{j}\right\|^{2}}{2 \sigma_{j}^{2}}\right), j=1,2, \ldots, m,
$$

where $\boldsymbol{x}$ is the input vector, $c_{i}$ is the center of the $i$-th a basis function with the same dimension vector of $\boldsymbol{x}$, and $\sigma_{j}$ is the width of the Gauss basis function of the $j$-th neurons in the hidden layer and is greater than zero. $m$ is the number of hidden layer nodes.

RBF network weight vector $\boldsymbol{w}=\left[w_{1}, w_{2}, \ldots, w_{m}\right]^{\mathrm{T}}$.

Output layer consists of six neurons, the output of the adjusted TDOA value. The output of the RBF network can be expressed as

$$
y(t)=\boldsymbol{w}^{\mathrm{T}} \boldsymbol{\varphi}=w_{1} \varphi_{1}+w_{2} \varphi_{2}+\ldots+w_{m} \varphi_{m} .
$$

\subsection{Adaptive RBF network}

The pure RBF network limits the number of hidden layer nodes, and then according to the network output error in gradient descent method the network weights and the center of the hidden layer neurons vector is adjusted, to achieve ultimately a predetermined error indicators as a condition of the end.

Learning algorithm in reference [6,7], adaptive RBF network does not need to determine the number of hidden layer nodes, but in the process of learning, according to the distribution of the errors in the input space, increase the number of hidden layer nodes adaptively. Make sure that the network approximation precision are higher, and through the comprehensive evaluation of the contributions of RBF network, adaptive to remove small contributions of RBF, to maintain a better generalization ability, finally in the case of both has good performance to achieve the effect where a network structure is simple. 
Adding a policy necessitates counting the output of the error of each input vector, and find large point error relatively with comparing, then inserted into the hidden layer nodes appropriately.

Set $\left(x_{k}, y_{k}\right), k=1,2, \ldots, N$, is a set of training samples, in the initial time, the number of hidden layer nodes is zero, each adding operation, according to the following guidelines determine whether add the hidden layer nodes:

$$
\begin{gathered}
e_{k}=\left|\boldsymbol{y}_{k}-f\left(\boldsymbol{x}_{k}\right)\right|>e, \\
\left\|\boldsymbol{x}_{k}-\boldsymbol{c}_{k, \text { nearest }}\right\|>\frac{1}{2}\left\|\boldsymbol{x}_{k}-\boldsymbol{x}_{k, \text { nearest }}\right\|,
\end{gathered}
$$

where $e=\sqrt{\frac{1}{N} \sum_{i=1}^{N} e_{i}^{2}}$ is the output mean square error of the network; $\boldsymbol{c}_{k \text {,nearest }}$ and $\boldsymbol{x}_{k, \text { nearest }}$ are respectively corresponding to the center of hidden layer nodes and the input vector, which is the most close to the input vector. If you meet the adding conditions, $\left(\boldsymbol{x}_{k}+\boldsymbol{x}_{k \text {, nearest }}\right) / 2$ is set to the new center of hidden layer nodes, and $e_{k}$ is set as the new node weights.

Deletion policy is based on each hidden layer nodes of the network with the contribution of different sizes. Big node will be retained, and small contribution nodes will be removed. For any hidden layer nodes $i, A_{i}$ used to show its contribution to the whole network. Defined $A_{i}$ as

$$
A_{i}=\sum_{k=1}^{N}\left|\frac{w_{i} h_{i}\left(\left\|\boldsymbol{x}_{k}-\boldsymbol{c}_{i}\right\|\right)}{\boldsymbol{y}_{k}}\right| .
$$

Before deletion operation on the normalized processing $\tilde{A}_{l}=A_{i} / A_{\max }$. If the final judgment rule for: $\tilde{A}_{l}<\theta$, the $i$-th hidden layer nodes are removed, and $\theta$ is decision threshold.

Before the RBF training, determine the maximum number $M$ and permissible error $E_{r}$, as a condition of the training end. The process includes two parts: I. A gradient descent method is used to adjust the center of the hidden layer nodes and weights between hidden layer and output layer, and the corresponding is adjusted for each cycle. II. It is to perform add or delete operation, with the method of interval to perform add or delete operations, avoiding excessive fitting operation on network and excessive deleting nodes.

It can adaptively adjust the parameters of the hidden layer nodes and the number, so more flexibility and applicability than fixed nodes number of RBF neural network.

\section{Algorithm process simulation and the analysis of simulation results}

\subsection{Algorithm process}

According to the above deduction, can get a general process of the algorithm in Fig. 1. 


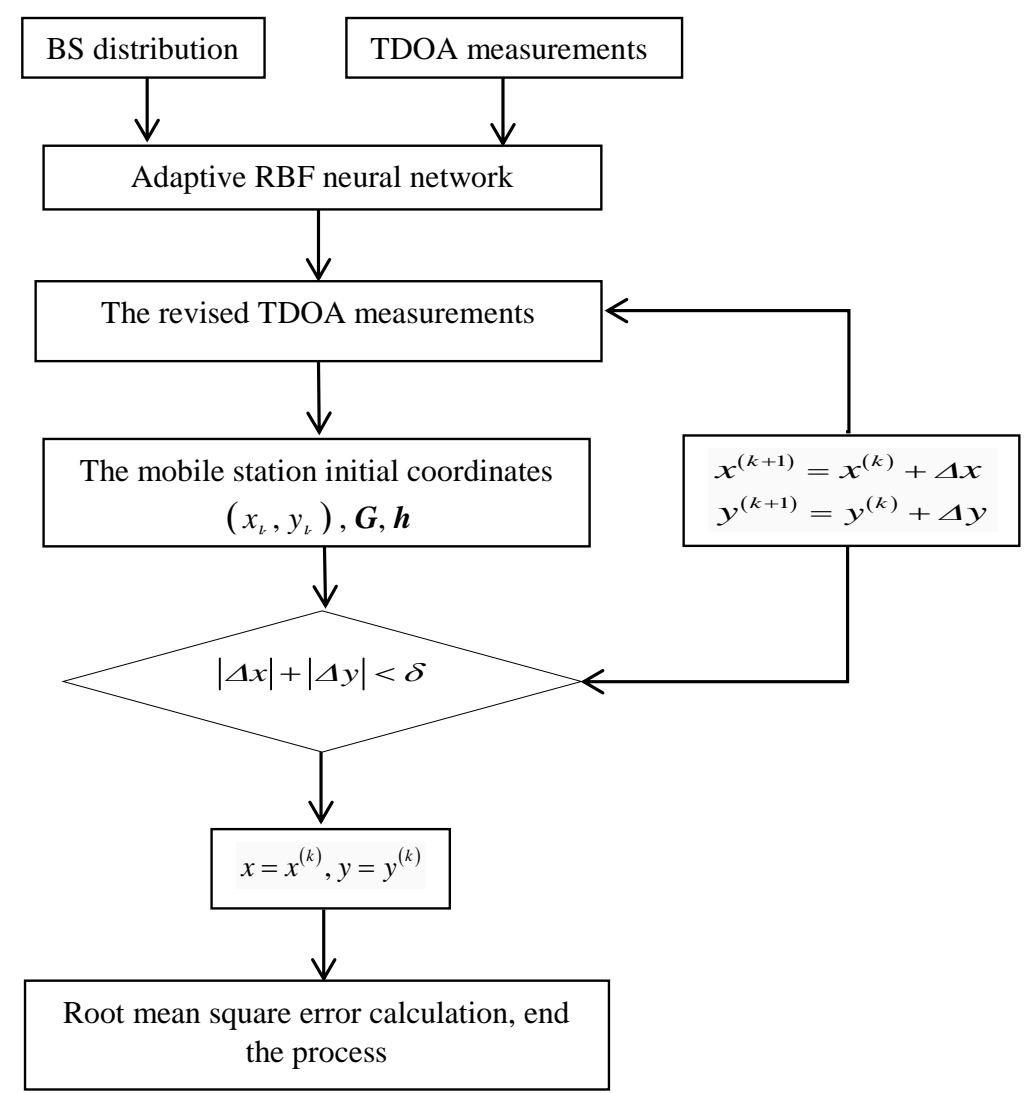

Fig. 1. The flow chart of the proposed algorithm

\subsection{The algorithm simulation}

To illustrate and validate performances of the algorithm on the positioning accuracy under NLOS, the proposed algorithm is compared with Chan algorithm and leastsquare algorithm (LS), Taylor series expansion algorithm [9], the Taylor series expansion positioning algorithm based on least square (LS) respectively on the condition of comparative analysis. At the same time, it is also compared with the performance of pure RBF network.

Based on seven typical cellular network structures, location coordinates are for the base station $\quad \mathrm{BS}_{1}(0,0), \quad \mathrm{BS}_{2}(0, \sqrt{3} R), \quad \mathrm{BS}_{3}(3 R / 2, \sqrt{3} R / 2), \quad \mathrm{BS}_{4}(3 R / 2,-\sqrt{3} R / 2)$, $\mathrm{BS}_{5}(0,-\sqrt{3} R), \mathrm{BS}_{6}(-3 R / 2, \sqrt{3} R / 2), \mathrm{BS}_{7}(-3 R / 2, \sqrt{3} R / 2)$. As the service stations are located in the central area of the base station, district radius $R$ is $2000 \mathrm{~m}$, initial coordinate is for the mobile station $(1000 \mathrm{~m}, 1000 \mathrm{~m})$, convergence threshold $\delta=10 \mathrm{~m}$. Simulation, using Monte Carlo simulation, simulation run independently each 1000 times, and the simulation of measuring $d_{i}=r_{i}+r_{i}^{\text {noise }}+r_{i}^{\text {nlos }}$, where $r_{i}^{\text {noise }}$ is the measuring error, generally can be thought of zero mean Gaussian distribution. $r_{i}^{\text {nlos }}$ is the NLOS error, which is 
evenly distributed between 0 and MAX random variables [10], including MAX is NLOS error factor, in order to determine a value. MAX size must affect the positioning accuracy of positioning algorithm, with MAX size being between 100 and $600 \mathrm{~m}$. The TDOA system measurement error are assumed to be independent identically zero mean Gaussian distribution. For the convenience, the TDOA measurements transform into ranging error, which is the product of TDOA measurement error and the speed of light. Because only increased the product of constant term, to the actual error distribution, which won't impact, and between MS and BS are NLOS. Specific simulation process is: first of all, according to the selection of MS coordinates and TDOA measurements of measurement error model, produced the corresponding simulation; Secondly, based on adaptive RBF network training, with MS excluding NLOS error of TDOA for target sample vector on the network was trained; Finally, using the trained RBF network to simulate the TDOA measurements, estimated position with the adjusted TDOA value using Taylor series expansion algorithm.

Positioning accuracy is a key indicator to measure the effectiveness of the algorithm, with the root mean square error RMSE $=\sqrt{\left(x-x_{0}\right)^{2}+\left(y-y_{0}\right)^{2}}$ for location accuracy, where $(x, y)$ is for MS estimated position, and $\left(x_{0}, y_{0}\right)$ is the MS estimated location.

\subsection{The results of simulation analysis}

In Fig. 2, the effect of base station number is illustrated. The increase of $\mathrm{N}$ greatly improves the performance of the proposed method as the curve shows. This is because the increase of the number of base station makes the increase of the redundant information, so that the positioning performance was improved to illustrate Proposed Algorithm is not sensitive to number of base stations. Look from the positioning effect, under different number of base stations, performance of Proposed Algorithm is better than other algorithms.

Fig. 3 shows, that with the increase of NLOS error $\delta_{\text {NLOS }}$, a sharp rise in the positioning error of Chan Algorithm, LS Algorithm and Taylor Algorithm can be observed. Proposed Algorithm under the influence of NLOS error $\delta_{\text {NLOS }}$ is minimal and has the highest positioning accuracy than other algorithms. When the number of NLOS error $\delta_{\mathrm{NLOS}}$ is $500 \mathrm{~m}$, Proposed Algorithm positioning accuracy compared with Chan Algorithm, LS Algorithm, Taylor Algorithm, LS-Taylor Algorithm and RBF-Taylor Algorithm is increased by $83.41 \%, 76.74 \%, 73.80 \%, 45.08 \%$ and $38.84 \%$ respectively. Proposed algorithm in this paper shows its own advantages, in the premise of guaranteing the positioning accuracy is better than other algorithms, and as environmental deterioration in the channel, the positioning of the algorithm performance degradation speed is lower than other algorithms, which shows that adaptive RBF network of NLOS error correction effectively inhibit the increase of the positioning error, and make it more stable. 


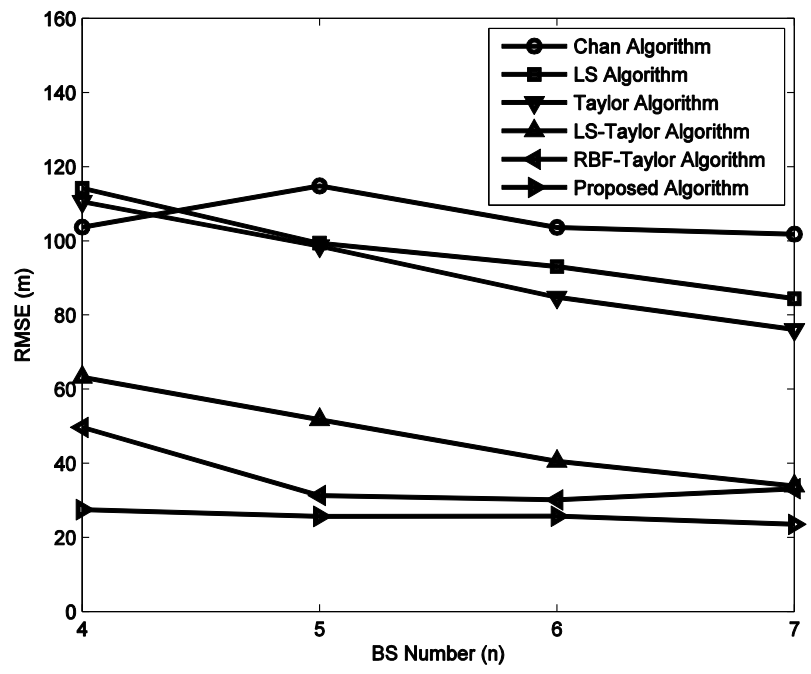

Fig. 2. RMSE performance comparison of positioning Algorithms on different number of base station

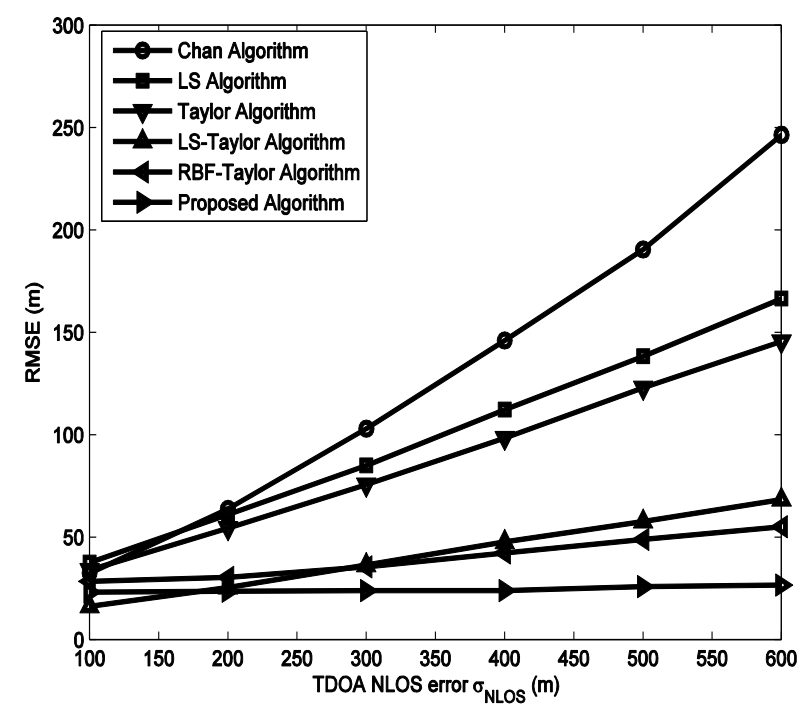

Fig. 3. RMSE performance comparison of positioning Algorithms on NLOS error

In Fig. 4, with the increase of cell radius increase, positioning error is less probability within $125 \mathrm{~m}$. The reason behind this is the increase of NLOS error with the increase of distance. It is because of cell radius increasing, the distance between the MS and BS will increase, so lead to the increase of the NLOS error, decline of the positioning accuracy. Positioning performance of the proposed algorithm is superior to the other three algorithms, this is because the algorithm of TDOA measurements revised, eliminated the influence of NLOS error, to a certain extent and thus improve the positioning accuracy. 


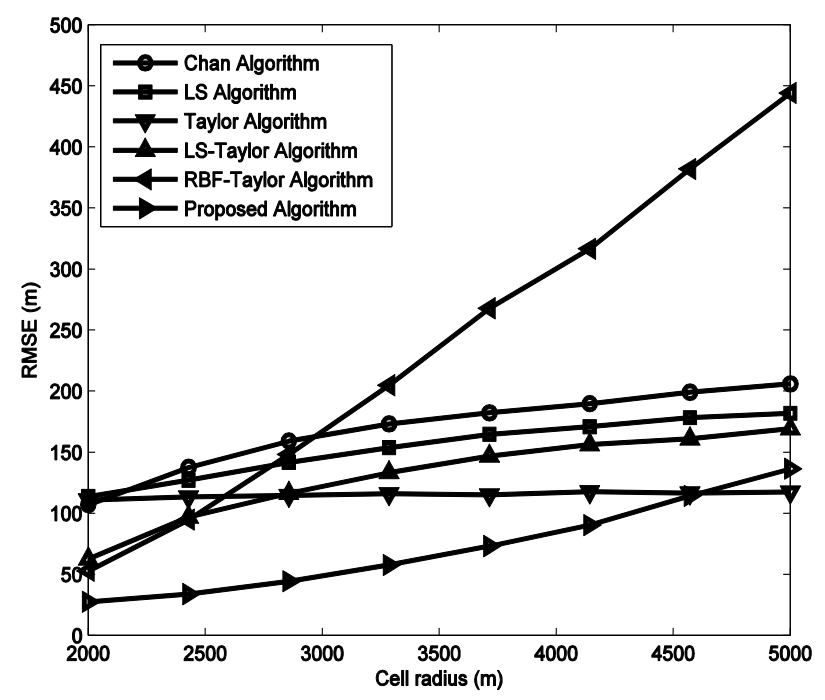

Fig. 4. RMSE performance comparison of positioning Algorithms on NLOS error

Fig. 5 shows the number of hidden layer nodes for RBF-Taylor Algorithm and Proposed Algorithm on different NLOS error $\delta_{\text {NLOS }}$ under Measurement noise standard deviation $\delta$ LOS is $30 \mathrm{~m}$. From Fig. 5, comparing the number of hidden layer nodes which reach the RBF training permissible error threshold need, Proposed Algorithm is less than RBF-Taylor Algorithm obviously. Therefore, nodes loss of Proposed Algorithm is better than RBF-Taylor Algorithm under the condition of NLOS.

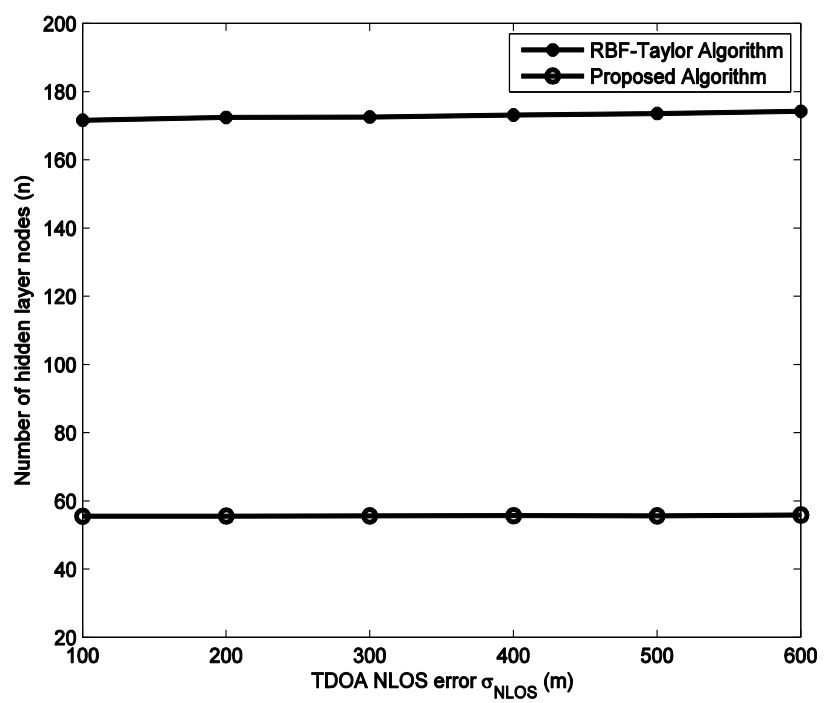

Fig. 5. Number of hidden layer nodes for RBF-Taylor Algorithm and Proposed Algorithm on NLOS error 


\section{Conclusions}

The NLOS error is a difficulty found in the study of wireless positioning system, as the channel environment deteriorating, the NLOS error will increase, so the positioning performance of the algorithm has a certain decline. This paper proposed Taylor-series expansion location algorithm, based on self-adaptive RBF neural network algorithm. By the simulation, the proposed algorithm has high location accuracy, reliability of the positioning performance and low consumption of hidden layer nodes. The result from the nalysis shows that the proposed algorithm is suitable for complicated multipath environment.

Acknowledgements: This work was supported by 2013 Technology Foundation for Selected Overseas Chinese Scholar (No 401053740756), Ministry of Personnel of Beijing and The Scientific Research Foundation for the Returned Overseas Chinese Scholars, State Education Ministry and Training program for Outstanding Young Scholars (No 14085).

\section{References}

1. Winternitz, L. M. B., W. A. B a m ford, G. W. Heckler. A GPS Receiver for HighAltitude Satellite Navigation. - IEEE J. Selected Topics in Signal Processing, Vol. 3, 2009, No 4, pp. 541-556.

2. Soltanian, M., A. M. Pezeshk, A. Mahdavi. A New Iterative Position Finding Algorithm Based on Taylor Series Expansion. - In: Proc. of 19th Iranian Conference on Electrical Engineering (ICEE’2011), 2011, 17-19 May, Tehran, Iran, pp. 1-4.

3. Y u, X. J., W. W e i, Z. Z. Li a n g. A New TDOA Location Technique Based on Taylor Series Expansion in Cellular Networks. - In: Proc. of 4th International Conference on Parallel and Distributed Computing, Applications and Technologies, 27-29 August 2003, Chengdu, China, pp. 378-381.

4. Hu a, T. G., D. Jing, N. C. Ji a n. Series Type of Neural Network and Its Application and Research in Forecasting of River Floods. - Research of Water Resources, Vol. 24, 2003, No 2 .

5. Y u n, C. X., M. Q i an g, T. A 1 kh a robi. New Neural Networks Based on Taylor Series and their Research. - In: Proc. of 2nd IEEE International Conference on Computer Science and Information Technology, 8-11 August 2009, Beijing, China, pp. 291-194.

6. B i a o, W. L., F. J i a n. Study of Self-Adaptive RBF Neural Network Control Method for the Engine Idle Speed Control. - In: Proc. of 2011 International Conference on Consumer Electronics, Communications and Networks (CECNet), 16-18 April 2011, XianNing, China, pp. 2633-2636.

7. M e n g, K., Z. Y. D on g, D. H. W a n g, K. P. W on g. A Self-Adaptive RBF Neural Network Classifier for Transformer Fault Analysis. - IEEE Transactions on Power Systems, Vol. 25, 2010, No 3, pp. 1350-1360.

8. S in g h, P., S. A g r a w a l. TDOA Based Node Localization in WSN Using Neural Networks. In: Proc. of 2013 International Conference on Communication Systems and Network Technologies (CSNT), 6-8 April 2013, Gwalior, India, pp. 400-404.

9. K e g e n, Y., Y. J. Gu o, I. O p p e r m a n n. Modified Taylor Series Expansion Based Positioning Algorithms. - In: Proc. of IEEE Vehicular Technology Conference, 11-14 May 2008, Singapore, pp. 2656-2660.

10. T o n g, C. Y., T. W. Y u e, S. H. Ch e u n g, C. P. C h u n g. Time-of-Arrival Based Localization under NLOS Conditions. - IEEE Transactions on Vehicular Technology, Vol. 55, 2006, No 1, pp. 17-24. 